\title{
On the parametrization of boundary-value problems with three-point non-linear restrictions
}

\author{
Miklós Rontó and K. Marynets
}




\title{
ON THE PARAMETRIZATION OF BOUNDARY-VALUE PROBLEMS WITH THREE-POINT NON-LINEAR RESTRICTIONS
}

\author{
MIKLÓS RONTÓ AND K. MARYNETS
}

Received 19 December, 2011

\begin{abstract}
We obtain some results concerning the solutions of certain types of three-point nonlinear boundary-value problems, subject to non-linear boundary conditions. We reduce the given problem to a parametrized one with linear two-point boundary restrictions containing some artificially introduced parameters. By the study of the transformed two-point problem we justify our method, which is based upon a special type of approximations constructed in analytic form.
\end{abstract}

2000 Mathematics Subject Classification: 34B15; 34B08

Keywords: three-point non-linear boundary-value problem, successive approximations, numericalanalytic method, determining system

\section{INTRODUCTION}

The non-linear three-point boundary-value problems were studied earlier mostly in the case of linear boundary restrictions. They were investigated in [2],[4], and [5] by using parametrization and the so-called numerical analytic method based upon successive approximations [7].

According to the basic idea of the above mentioned method, at first the original boundary-value problem is transformed to a two-point one which is replaced by the problem for the "perturbed" differential equation containing some new artificially introduced parameters, whose numerical values should be determined. The solutions of the parametrized problem are built in analytic form by successive iterations with all iterations depending upon the artificially introduced parameters.

Here we give a possible approach to transform a three-point boundary-value problem with non-linear boundary restrictions into a two-point one with linear boundary conditions using ideas from $[1,3,5]$. 


\section{Problem SETting}

We consider the three-point boundary-value problem subject to the nonlinear boundary conditions

$$
\begin{gathered}
\frac{d x(t)}{d t}=f(t, x(t)), t \in[0, T], x \in \mathbb{R}^{n}, \\
g\left(x(0), x\left(t_{1}\right), x(T)\right)=0, t_{1} \in(0, T) .
\end{gathered}
$$

Here, we suppose that the functions

$$
f:[0, T] \times D \rightarrow \mathbb{R}^{n}
$$

and

$$
g: D \times D \times D \rightarrow \mathbb{R}^{n},(n \geq 2)
$$

are continuous, where $D \subset \mathbb{R}^{n}$ is a closed and bounded domain.

The problem is the determination of a continuously differentiable solution of the system of differential equations (2.1) satisfying non-linear three-point boundary restrictions (2.2).

\section{CONSTRUCTION OF AN EQUIVALENT PROBLEM WITH LINEAR TWO-POINT BOUNDARY CONDITIONS}

To pass to the linear two-point boundary conditions from (2.2), we replace the values of the components of the solution of (2.1), (2.2) at the points $t=0, t=t_{1}$ and $t=T$ by the following parameters:

$$
\begin{gathered}
z:=x(0)=\operatorname{col}\left(x_{1}(0), x_{2}(0), \ldots, x_{n}(0)\right)=\operatorname{col}\left(z_{1}, z_{2}, \ldots, z_{n}\right), \\
\eta:=x\left(t_{1}\right)=\operatorname{col}\left(x_{1}\left(t_{1}\right), x_{2}\left(t_{1}\right), \ldots, x_{n}\left(t_{1}\right)\right)=\operatorname{col}\left(\eta_{1}, \eta_{2}, \ldots, \eta_{n}\right), \\
\lambda:=x(T)=\operatorname{col}\left(x_{1}(T), x_{2}(T), \ldots, x_{n}(T)\right)=\operatorname{col}\left(\lambda_{1}, \lambda_{2}, \ldots, \lambda_{n}\right) .
\end{gathered}
$$

Let us rewrite the boundary conditions (2.2) in the form:

$$
A x(0)+C x(T)+g\left(x(0), x\left(t_{1}\right), x(T)\right)=A x(0)+C x(T),
$$

where $A$ is some given matrix and $C \equiv I_{n}$, where $I_{n}$ is a unit $n \times n$ matrix.

Using parametrization (3.1), the non-linear three-point boundary restrictions (3.2) can be written as a two-point ones:

$$
A x(0)+x(T)=A z+\lambda-g(z, \eta, \lambda) .
$$

Let us put

$$
d(z, \eta, \lambda):=A z+\lambda-g(z, \eta, \lambda) .
$$

Taking into account (3.4), the parametrized boundary conditions (3.3) can be rewritten in the form:

$$
A x(0)+x(T)=d(z, \eta, \lambda) .
$$


Let us consider the special case of (3.5) where we take the zero matrix instead of $A$ :

$$
\begin{gathered}
x(0)=z, \\
x(T)=d(z, \eta, \lambda) .
\end{gathered}
$$

So, instead of the original three-point boundary-value problem with non-linear boundary conditions (2.1), (2.2) we study an equivalent parametrized two-point one, containing linear separated boundary restrictions (2.1), (3.6).

Remark 1. The set of the solutions of the non-linear three-point boundary-value problem (2.1), (2.2) coincides with the set of the solutions of the two-point problem (2.1), (3.6) satisfying additional conditions (3.1).

\section{CONSTRUCTION OF THE SUCCESSIVE APPROXIMATIONS}

Let us introduce the vector

$$
\delta_{D}(f):=\frac{1}{2}\left[\max _{(t, x) \in[0, T] \times D} f(t, x)-\min _{(t, x) \in[0, T] \times D} f(t, x)\right] .
$$

The original boundary-value problem $(2.1),(2.2)$ is such that the subset

$$
D_{\beta}:=\left\{z \in D: B\left(z, \max _{t \in[0, T]}\left|z+\frac{t}{T}[d(z, \eta, \lambda)-z]\right|\right) \subset D, \forall \eta, \lambda \in D\right\}
$$

is non-empty

$$
D_{\beta} \neq \varnothing \text {. }
$$

Assume that the function $f(t, x)$ satisfies Lipschitz condition of the form

$$
|f(t, u)-f(t, v)| \leq K|u-v|,
$$

for all $t \in[0, T],\{u, v\} \subset D$ with some non-negative constant matrix $K=\left(k_{i j}\right)_{i, j=1}^{n}$.

Moreover, we suppose that the spectral radius $r(K)$ of the matrix $K$ satisfies the following inequality

$$
r(K)<\frac{10}{3 T}
$$

Let us associate to the parametrized boundary-value problem (2.1), (3.6) the sequence of functions:

$$
\begin{aligned}
x_{m}(t, z, \eta, \lambda):=z+ & \int_{0}^{t} f\left(s, x_{m-1}(s, z, \eta, \lambda)\right) d s- \\
& -\frac{t}{T} \int_{0}^{T} f\left(s, x_{m-1}(s, z, \eta, \lambda)\right) d s+\frac{t}{T}[d(z, \eta, \lambda)-z],
\end{aligned}
$$

where $m=1,2,3, \ldots$

$$
x_{0}(t, z, \eta, \lambda)=z+\frac{t}{T}[d(z, \eta, \lambda)-z] \in D_{\beta},
$$


$\eta=\operatorname{col}\left(\eta_{1}, \eta_{2}, \ldots, \eta_{n}\right) \in D, \lambda=\operatorname{col}\left(\lambda_{1}, \lambda_{2}, \ldots, \lambda_{n}\right) \in D$,

$x_{m}(t, z, \eta, \lambda)=\operatorname{col}\left(x_{m, 1}(t, z, \eta, \lambda), x_{m, 2}(t, z, \eta, \lambda), \ldots, x_{m, n}(t, z, \eta, \lambda)\right)$ and $z, \eta, \lambda$ are considered as parameters.

It is easy to check that the functions $x_{m}(t, z, \eta, \lambda)$ satisfy linear parametrized boundary conditions (3.6) for all $m \geq 1, z \in D_{\beta}, \eta \in D, \lambda \in D$.

The following statement establishes the convergence of the sequence (4.5).

Theorem 1. Assume that the function $f:[0, T] \times D \rightarrow \mathbb{R}^{n}$ on the right side of the system of differential equations (2.1) and the parametrized boundary restrictions (3.6) satisfy conditions (4.2)-(4.4).

Then for all fixed $z \in D_{\beta}, \eta \in D, \lambda \in D$ :

(1) The functions of the sequence (4.5) are continuously differentiable and satisfy the parametrized boundary conditions (3.6):

$$
\begin{gathered}
x_{m}(0, z, \eta, \lambda)=z, \\
x_{m}(T, z, \eta, \lambda)=d(z, \eta, \lambda),
\end{gathered}
$$

$m=1,2,3, \ldots$

(2) The sequence of functions (4.5) for $t \in[0, T]$ converges uniformly as $m \rightarrow \infty$ to the limit function

$$
x^{*}(t, z, \eta, \lambda)=\lim _{m \rightarrow \infty} x_{m}(t, z, \eta, \lambda) .
$$

(3) The limit function $x^{*}(t, z, \eta, \lambda)$ satisfies the parametrized linear two-point boundary conditions:

$$
\begin{gathered}
x^{*}(0, z, \eta, \lambda)=z, \\
x^{*}(T, z, \eta, \lambda)=d(z, \eta, \lambda) .
\end{gathered}
$$

(4) The limit function (4.8) for all $t \in[0, T]$ is a unique continuously differentiable solution of the integral equation

$$
x(t)=z+\int_{0}^{t} f(s, x(s)) d s-\frac{t}{T} \int_{0}^{T} f(s, x(s)) d s+\frac{t}{T}[d(z, \eta, \lambda)-z],
$$

i. e. it is the solution of the Cauchy problem for the modified system of differential equations:

$$
\begin{gathered}
\frac{d x}{d t}=f(t, x)+\Delta(z, \eta, \lambda), \\
x(0)=z,
\end{gathered}
$$

where

$$
\Delta(z, \eta, \lambda):=\frac{1}{T}[d(z, \eta, \lambda)-z]-\frac{1}{T} \int_{0}^{T} f(s, x(s)) d s .
$$


(5) The following error estimate holds:

$$
\left|x^{*}(t, z, \eta, \lambda)-x_{m}(t, z, \eta, \lambda)\right| \leq \frac{20}{9} t\left(1-\frac{t}{T}\right) Q^{m}\left(I_{n}-Q\right)^{-1} \delta_{D}(f),
$$

where matrix

$$
Q:=\frac{3 T}{10} K
$$

Proof. We will prove that the sequence of functions (4.5) is a Cauchy sequence in the Banach space $C\left([0, T], \mathbb{R}^{n}\right)$. First we show that $x_{m}(t, z, \eta, \lambda) \in D$, for all $(t, z, \eta, \lambda) \in[0, T] \times D_{\beta} \times D \times D, m \in \mathbb{N}$.

Indeed, using the estimates of Lemma 2.3 from [7] (see also Lemma 3 [6] and Lemma $2[4])$ :

$$
\left|\int_{0}^{t}\left[f(\tau)-\frac{1}{T} \int_{0}^{T} f(s) d s\right] d \tau\right| \leq \frac{1}{2} \alpha_{1}(t)\left[\max _{t \in[0 . T]} f(t)-\min _{t \in[0, T]} f(t)\right],
$$

where

$$
\alpha_{1}(t)=2 t\left(1-\frac{t}{T}\right),\left|\alpha_{1}(t)\right| \leq \frac{T}{2}, t \in[0, T],
$$

relation (4.5) for $m=0$ implies that:

$$
\begin{aligned}
& \left|x_{1}(t, z, \eta, \lambda)-x_{0}(t, z, \eta, \lambda)\right| \leq \\
& \leq\left|\int_{0}^{t}\left[f(t, z)-\frac{1}{T} \int_{0}^{T} f(s, z) d s\right] d t\right| \leq \\
& \quad \leq \alpha_{1}(t) \delta_{D}(f) \leq \frac{T}{2} \delta_{D}(f) .
\end{aligned}
$$

Therefore, by virtue of (4.17), we conclude that $x_{1}(t, z, \eta, \lambda) \in D$ whenever $(t, z, \eta, \lambda) \in[0, T] \times D_{\beta} \times D \times D$.

By induction we can easily establish that all functions (4.5) are also contained in the domain $D \forall m=1,2,3, \ldots, t \in[0, T], z \in D_{\beta}, \eta \in D, \lambda \in D$.

Now, consider the difference of functions:

$$
\begin{aligned}
x_{m+1}(t, z, \eta, \lambda) & -x_{m}(t, z, \eta, \lambda)= \\
= & \int_{0}^{t}\left[f\left(s, x_{m}(s, z, \eta, \lambda)\right)-f\left(s, x_{m-1}(s, z, \eta, \lambda)\right)\right] d s- \\
& \quad-\frac{t}{T} \int_{0}^{T}\left[f\left(s, x_{m}(s, z, \eta, \lambda)\right)-f\left(s, x_{m-1}(s, z, \eta, \lambda)\right)\right] d s,
\end{aligned}
$$

$m=1,2,3, \ldots$ and introduce the notation:

$$
r_{m}(t, z, \eta, \lambda):=\left|x_{m}(t, z, \eta, \lambda)-x_{m-1}(t, z, \eta, \lambda)\right|, m=1,2,3, \ldots
$$

By virtue of the estimation (4.15) and of the Lipschitz condition (4.3), we have: 


$$
\begin{aligned}
r_{m+1}(t, z, \eta, \lambda) \leq K\left[\left(1-\frac{t}{T}\right) \int_{0}^{t} r_{m}(s, z, \eta, \lambda) d s+\right. & \\
& \left.+\frac{t}{T} \int_{t}^{T} r_{m}(s, z, \eta, \lambda) d s\right],
\end{aligned}
$$

$\forall m=0,1,2, \ldots$

According to (4.17)

$$
r_{1}(t, z, \eta, \lambda)=\left|x_{1}(t, z, \eta, \lambda)-x_{0}(t, z, \eta, \lambda)\right| \leq \alpha_{1}(t) \delta_{D}(f) .
$$

By virtue of the statement of Lemma 3 [4] of the form

$$
\alpha_{m+1}(t) \leq \frac{10}{9}\left(\frac{3}{10} T\right)^{m} \alpha_{1}(t), m=0,1,2, \ldots
$$

obtained for sequence of functions

$$
\begin{gathered}
\alpha_{m+1}(t)=\left(1-\frac{t}{T}\right) \int_{0}^{t} \alpha_{m}(s) d s+\frac{t}{T} \int_{t}^{T} \alpha_{m}(s) d s, m=0,1,2, \ldots \\
\alpha_{0}(t)=1, \alpha_{1}(t)=2 t\left(1-\frac{t}{T}\right)
\end{gathered}
$$

from (4.19) for $m=1$ follows:

$$
\begin{aligned}
& r_{2}(t, z, \eta, \lambda) \leq K \delta_{D}(f)\left[\left(1-\frac{t}{T}\right) \int_{0}^{t} \alpha_{1}(s) d s+\frac{t}{T} \int_{t}^{T} \alpha_{1}(s) d s\right] \leq \\
& \leq K \alpha_{2}(t) \delta_{D}(f) .
\end{aligned}
$$

By induction using (4.22), we can easily obtain that

$$
r_{m+1}(t, z, \eta, \lambda) \leq K^{m} \alpha_{m+1}(t) \delta_{D}(f),
$$

$m=0,1,2, \ldots$, where $\alpha_{m+1}(t), \alpha_{m}(t)$ are calculated according to (4.22), and $\delta_{D}(f)$ is given by (4.1).

By virtue of the estimate (4.21) from (4.23) we have

$$
r_{m+1}(t, z, \eta, \lambda) \leq \frac{10}{9} \alpha(t)\left[Q^{m} \delta_{D}(f)+K Q^{m-1}|d(z, \eta, \lambda)-z|\right],
$$

$\forall m=1,2,3, \ldots$, where the matrix $Q$ is given by (4.14).

Therefore, in view of (4.24)

$$
\begin{gathered}
\left|x_{m+j}(t, z, \eta, \lambda)-x_{m}(t, z, \eta, \lambda)\right| \leq\left|x_{m+j}(t, z, \eta, \lambda)-x_{m+j-1}(t, z, \eta, \lambda)\right|+ \\
+\left|x_{m+j-1}(t, z, \eta, \lambda)-x_{m+j-2}(t, z, \eta, \lambda)\right|+\ldots+ \\
+\left|x_{m+1}(t, z, \eta, \lambda)-x_{m}(t, z, \eta, \lambda)\right|= \\
=\sum_{i=1}^{j} r_{m+i}(t, z, \eta, \lambda) \leq \frac{10}{9} \alpha_{1}(t) \sum_{i=1}^{j} Q^{m+i} \delta_{D}(f)= \\
=\frac{10}{9} \alpha_{1}(t) Q^{m} \sum_{i=0}^{j-1} Q^{i} \delta_{D}(f) .
\end{gathered}
$$


Since, due to the condition (4.4), the maximum eigenvalue of the matrix $Q$ of the form (4.14) does not exceed the unity, we have

$$
\sum_{i=0}^{j-1} Q^{i} \leq\left(I_{n}-Q\right)^{-1}, \lim _{m \rightarrow \infty} Q^{m}=O_{n},
$$

where $O_{n}$ is a zero $n \times n$ matrix.

Therefore we conclude from (4.25) that, according to the Cauchy criterium, the sequence $\left\{x_{m}(t, z, \eta, \lambda)\right\}$ of the form (4.5) uniformly converges in the domain $(t, z, \eta, \lambda) \in[0, T] \times D_{\beta} \times D \times D$ to the limit function $x^{*}(t, z, \eta, \lambda)$. Since all functions $x_{m}(t, z, \eta, \lambda)$ of the sequence (4.5) satisfy the boundary conditions (3.6) for all values of the artificially introduced parameters, the limit function $x^{*}(t, z, \eta, \lambda)$ also satisfies these conditions. Passing to the limit as $m \rightarrow \infty$ in equality (4.5) we show that the limit function satisfies both the integral equation (4.9) and the Cauchy problem (4.10), (4.11), where $\Delta(z, \eta, \lambda)$ is given by (4.12).

Consider the Cauchy problem

$$
\begin{gathered}
\frac{d x}{d t}=f(t, x)+\mu, t \in[0, T] \\
x(0)=z,
\end{gathered}
$$

where $\mu=\operatorname{col}\left(\mu_{1}, \ldots, \mu_{n}\right)$ is a control parameter.

Theorem 2. Under the conditions of Theorem 1 , the solution $x=x(\cdot, z, \eta, \lambda, \mu)$ of the initial value problem (4.26), (4.27) satisfies the boundary conditions (3.6) if and only if $x=x(\cdot, z, \eta, \lambda, \mu)$ coincides with the limit function $x^{*}(\cdot, z, \eta, \lambda, \mu)$ of the sequence (4.5). Moreover

$$
\mu=\mu_{z, \eta, \lambda}=\frac{1}{T}[d(z, \eta, \lambda)-z]-\frac{1}{T} \int_{0}^{T} f\left(s, x^{*}(s, z, \eta, \lambda)\right) d s .
$$

Proof. Sufficiency. Let us suppose that $\mu$ on the right side of the system of differential equations (4.26) is given by (4.28). By virtue of Theorem 1, the limit function (4.8) of the sequence (4.5) is the unique solution of the boundary-value problem (4.26), (3.6) for fixed values of parameters $z, \eta$ and $\lambda$ when $\mu=\mu_{z, \eta, \lambda}$. Furthermore the limit function $x^{*}(t, z, \eta, \lambda, \mu)$ satisfies initial conditions (4.27), i.e. it is a solution of the Cauchy problem (4.26), (4.27) when $\mu=\mu_{z, \eta, \lambda}$.

Necessity. Let us fix an arbitrary $\bar{\mu} \in \mathbb{R}^{n}$ and assume that the initial value problem (4.29), (4.27):

$$
\frac{d x}{d t}=f(t, x)+\bar{\mu}, t \in[0, T]
$$

has a solution $\bar{x}(t)$, that satisfies the two-point linear boundary conditions (3.6). Then $\bar{x}(t)$ satisfies an integral equation:

$$
\bar{x}(t)=z+\int_{0}^{t} f(s, \bar{x}(s)) d s+\bar{\mu} t
$$


for all $t \in[0, T]$.

When $t=T$ from (4.30) we get:

$$
T \bar{\mu}=\bar{x}(T)-z-\int_{0}^{T} f(s, \bar{x}(s)) d s .
$$

Under the assumption $\bar{x}(t)$ satisfies boundary restrictions (3.6):

$$
\bar{x}(T)=d(z, \eta, \lambda),
$$

and the initial condition

$$
\bar{x}(0)=z
$$

Substituting (4.32) in (4.31) we get:

$$
\bar{\mu}=\frac{1}{T} d(z, \eta, \lambda)-\frac{1}{T} z-\frac{1}{T} \int_{0}^{T} f(s, \bar{x}(s)) d s .
$$

On the other hand, it is proved that the limit function $x^{*}(\cdot, z, \lambda, \mu)$ is the solution of the initial value problem (4.26), (4.27) for $\mu=\mu_{z, \eta, \lambda}$ given by formula (4.28) and satisfies boundary conditions (3.6).

By analogy

$$
\begin{gathered}
x^{*}(t, z, \eta, \lambda, \mu)=z+\int_{0}^{t} f\left(s, x^{*}(s, z, \eta, \lambda, \mu)\right) d s+\mu_{z, \eta, \lambda} t \\
T \mu_{z, \eta, \lambda}=x^{*}(T, z, \eta, \lambda, \mu)-z-\int_{0}^{T} f\left(s, x^{*}(s, z, \eta, \lambda, \mu)\right) d s \\
x^{*}(T, z, \eta, \lambda, \mu)=d(z, \eta, \lambda), \\
x^{*}(0, z, \eta, \lambda, \mu)=z .
\end{gathered}
$$

By virtue of (4.34)-(4.36) it is easy to get that

$$
\mu_{z, \eta, \lambda}=\frac{1}{T} d(z, \eta, \lambda)-\frac{1}{T} z-\frac{1}{T} \int_{0}^{T} f\left(s, x^{*}(s, z, \eta, \lambda, \mu)\right) d s .
$$

Substituting (4.33) in (4.30) and (4.37) in (4.34), we get that for all $t \in[0, T]$

$$
\begin{aligned}
\bar{x}(t)=z+\int_{0}^{t} f(s, \bar{x}(s)) d s-\frac{t}{T} \int_{0}^{T} f(s, \bar{x}(s)) d s+\frac{t}{T}[d(z, \lambda)-z], \\
x^{*}(t, z, \eta, \lambda, \mu)=z+\int_{0}^{t} f\left(s, x^{*}(s, z, \eta, \lambda, \mu)\right) d s- \\
-\frac{t}{T} \int_{0}^{T} f\left(s, x^{*}(s, z, \eta, \lambda, \mu)\right) d s+\frac{t}{T}[d(z, \eta, \lambda)-z] .
\end{aligned}
$$


Using Theorem $1 \bar{x}(t) \in D$ and $x^{*}(t, z, \eta, \lambda, \mu) \in D$. Taking into account (4.38), (4.39) it follows that

$$
\begin{aligned}
x^{*}(t, z, \eta, \lambda, \mu)-\bar{x}(t)= & \int_{0}^{t}\left[f\left(s, x^{*}(s, z, \eta, \lambda, \mu)\right)-f(s, \bar{x}(s))\right] d s- \\
& -\frac{t}{T} \int_{0}^{T}\left[f\left(s, x^{*}(s, z, \eta, \lambda, \mu)\right)-f(s, \bar{x}(s))\right] d s .
\end{aligned}
$$

By the Lipschitz condition (4.3) from the relation (4.40), we get that the function

$$
\omega(t)=\left|x^{*}(t, z, \eta, \lambda, \mu)-\bar{x}(t)\right|, t \in[0, T]
$$

satisfies integral inequalities:

$$
\begin{aligned}
\omega(t) \leq K\left[\int_{0}^{t} \omega(s) d s+\frac{t}{T} \int_{0}^{T} \omega(s) d s\right] & \leq \\
& \leq K \alpha_{1}(t) \max _{s \in[0, T]} \omega(s), t \in[0, T],
\end{aligned}
$$

where $\alpha_{1}(t)$ is given by (4.16).

Using (4.42) recursively, we come to an inequality:

$$
\omega(t) \leq K^{m} \alpha_{m}(t) \max _{s \in[0, T]} \omega(s), t \in[0, T],
$$

where $m \in \mathbb{N}$ and functions $\alpha_{m}(t)$ are given by formula (4.22).

Taking into account (4.21), from (4.43) for each $m \in \mathbb{N}$ we get an estimation:

$$
\omega(t) \leq K \alpha_{1}(t) \frac{10}{9}\left(\frac{3 T}{10} K\right)^{m-1} \cdot \max _{s \in[0, T]} \omega(s), t \in[0, T] .
$$

By passing to the limit $m \rightarrow \infty$ in the last inequality and by virtue of (4.4), we come to the conclusion that

$$
\max _{s \in[0, T]} \omega(s) \leq Q^{m} \max _{s \in[0, T]} \omega(s) \rightarrow 0 .
$$

It means that the function $\bar{x}(t)$ coincides with $x^{*}(t, z, \eta, \lambda, \mu)$. Starting with (4.33) and (4.37), we come to the conclusion that $\bar{\mu}=\mu_{z, \eta, \lambda}$.

Let's find the relation of the limit function $x=x^{*}(t, z, \eta, \lambda)$ of the sequence (4.5) to the solution of the parametrized two-point linear boundary-value problem (2.1), (3.6) or to the equivalent three-point non-linear problem (2.1), (2.2).

Theorem 3. Assume that the conditions (4.2)-(4.4) are satisfied for the original boundary-value problem (2.1), (2.2).

Then the triplex $\left(x^{*}\left(\cdot, z^{*}, \eta^{*}, \lambda^{*}\right), \eta^{*}, \lambda^{*}\right)$ is the solution of the parametrized boundary-value problem (2.1), (3.6) if and only if $z^{*}=\left(z_{1}^{*}, z_{2}^{*}, \ldots, z_{n}^{*}\right)$, 
$\eta^{*}=\left(\eta_{1}^{*}, \eta_{2}^{*}, \ldots, \eta_{n}^{*}\right), \lambda^{*}=\left(\lambda_{1}^{*}, \lambda_{2}^{*}, \ldots, \lambda_{n}^{*}\right)$ satisfy a determining system of algebraic or transcendental equations

$$
\begin{array}{rl}
\Delta(z, \eta, \lambda)=\frac{1}{T}[d(z, \eta, \lambda)-z]-\frac{1}{T} \int_{0}^{T} & f\left(s, x^{*}(s, z, \eta, \lambda)\right) d s=0, \\
x^{*}\left(t_{1}, z, \eta, \lambda\right) & =\eta, \\
x^{*}(T, z, \eta, \lambda) & =\lambda .
\end{array}
$$

Proof. It suffices to apply Theorem 2 and to notice that the differential equation in (4.10) coincides with (2.1) if and only if triplex $\left(z^{*}, \eta^{*}, \lambda^{*}\right)$ satisfies an equation

$$
\Delta\left(z^{*}, \eta^{*}, \lambda^{*}\right)=0 .
$$

Taking into account (3.1) and the equivalence of (2.1), (2.2) and (2.1), (3.6), it is clear that $\left(x^{*}\left(\cdot, z^{*}, \eta^{*}, \lambda^{*}\right), \eta^{*}, \lambda^{*}\right)$ coincides with the solution of the parametrized boundary-value problem (2.1), (3.1), (3.6) if and only if $\left(x^{*}\left(\cdot, z^{*}, \eta^{*}, \lambda^{*}\right), \eta^{*}, \lambda^{*}\right)$ satisfies equations

$$
\begin{aligned}
& x^{*}\left(t_{1}, z, \eta^{*}, \lambda^{*}\right)=\eta^{*}, \\
& x^{*}\left(T, z, \eta^{*}, \lambda^{*}\right)=\lambda^{*} .
\end{aligned}
$$

It means that the triplex $\left(x^{*}\left(\cdot, z^{*}, \eta^{*}, \lambda^{*}\right), \eta^{*}, \lambda^{*}\right)$ is the solution of the parametrized boundary-value problem (2.1), (3.6) if and only if (4.45), (4.46), (4.47) are hold.

The next statement proves that the system of determining equations (4.45)- (4.47) defines all possible solutions of the original three-point boundary-value problem (2.1), (2.2).

Lemma 1. Let all conditions of Theorem 1 be satisfied. Furthemore there exist some vectors $z \in D_{\beta}, \eta \in D$ and $\lambda \in D$ that satisfy the system of determining equations (4.45)-(4.47).

Then the non-linear three-point boundary-value problem (2.1), (2.2) has solution $x(\cdot)$ such that:

$$
\begin{gathered}
x(0)=z, \\
x\left(t_{1}\right)=\eta, \\
x(T)=\lambda .
\end{gathered}
$$

Moreover this solution is given by formula

$$
x(t)=x^{*}(t, z, \eta, \lambda), t=[0, T],
$$

where $x^{*}(t, z, \eta, \lambda)$ is the limit function of the sequence (4.5). And if the boundaryvalue problem (2.1), (2.2) has a solution $x(\cdot)$, then this solution is given by (4.49), and the system of determining equations (4.45)-(4.47) is satisfied when

$$
\begin{aligned}
& z=x(0), \\
& \eta=x\left(t_{1}\right), \\
& \lambda=x(T) .
\end{aligned}
$$


Proof. We will apply Theorems 2 and 3. If there exist such $z \in D_{\beta}, \eta \in D$ and $\lambda \in$ $D$ that satisfy determining system (4.45), (4.46), (4.47), then according to Theorem 3 function (4.49) is a solution of the original boundary-value problem (2.1), (2.2). On the other hand, if $x(\cdot)$ is the solution of the original boundary-value problem (2.1), (2.2), then this function is the solution of the Cauchy problem (4.26), (4.27) for

$$
\begin{gathered}
\mu=0, \\
z=x(0) .
\end{gathered}
$$

As $x(\cdot)$ satisfies boundary restrictions (2.2) and equivalent conditions (3.5), by virtue of Theorem 2 an equality (4.49) is hold. Besides,

$$
\begin{gathered}
\mu=\mu_{z, \eta, \lambda}=0, \\
z=x(0),
\end{gathered}
$$

where vectors $\eta, \lambda$ are defined by (3.1).

But $\mu_{z, \eta, \lambda}$ is given by formula (4.28), that's why the first equation (4.45) of the determining system is satisfied, if

$$
\begin{gathered}
z=x(0), \\
\eta=x\left(t_{1}\right), \\
\lambda=x(T): \\
\Delta(z, \eta, \lambda)=0 .
\end{gathered}
$$

From (3.6) follows, that the next two equations (4.46), (4.47) of the determining system are hold, too. So we specified such triplexes $(z, \eta, \lambda)=\left(x(0), x\left(t_{1}\right), x(T)\right)$, that satisfy system of determining equations (4.45)-(4.47), that proves Lemma.

Remark 2. The main difficulty of the realization of this method is to find the limit function $x^{*}(\cdot, z, \eta, \lambda)$. But in most cases this problem can be solved using the properties of the approximate solution $x_{m}(\cdot, z, \eta, \lambda)$ built in an analytic form.

For $m \geq 1$ let us define the function $\Delta_{m}: D_{\beta} \times D \times D \rightarrow \mathbb{R}^{n}$ by formula

$$
\Delta_{m}(z, \eta, \lambda):=\frac{1}{T}[d(z, \lambda)-z]-\frac{1}{T} \int_{0}^{T} f\left(s, x_{m}(s, z, \eta, \lambda)\right) d s,
$$

where $z, \eta$ and $\lambda$ are given by the relation (3.1). To investigate the solubility of the parametrized boundary-value problem (2.1), (3.6) we observe an approximate determining system of algebraic or transcendental equations of the form

$$
\begin{array}{rl}
\Delta_{m}(z, \eta, \lambda)=\frac{1}{T}[d(z, \eta, \lambda)-z]-\frac{1}{T} \int_{0}^{T} & f\left(s, x_{m}(s, z, \eta, \lambda)\right) d s=0, \\
x_{m}\left(t_{1}, z, \eta, \lambda\right) & =\eta, \\
x_{m}(T, z, \eta, \lambda) & =\lambda,
\end{array}
$$

where $x_{m}(\cdot, z, \eta, \lambda)$ is a vector-function, that defines with the recursive relation (4.5). Increasing $m$, the systems (4.45)- (4.47) and (4.53), (4.54) are close enough to 
provide the needed precision of the approximate solution of the original boundaryvalue problem (2.1), (2.2).

\section{EXAMPLE}

Consider the system

$$
\left\{\begin{array}{l}
\frac{d x_{1}}{d t}=0.05 x_{2}-0.005 t^{2}+0.1=f_{1}\left(t, x_{1}, x_{2}\right), \\
\frac{d x_{2}}{d t}=-x_{2}^{2}+0.5 x_{1}+0.01 t^{4}+0.15 t=f_{2}\left(t, x_{1}, x_{2}\right),
\end{array}\right.
$$

where $t \in\left[0, \frac{1}{2}\right]$, with non-linear two-point boundary conditions

$$
\left\{\begin{array}{l}
g_{1}\left(x(0), x\left(\frac{1}{4}\right), x(1)\right):=x_{1}\left(\frac{1}{2}\right)+x_{2}^{2}(0)-x_{1}\left(\frac{1}{4}\right)-0.025=0, \\
g_{2}\left(x(0), x\left(\frac{1}{4}\right), x(1)\right):=x_{1}(0)+x_{2}\left(\frac{1}{2}\right)-x_{2}(0)-0.025=0 .
\end{array}\right.
$$

It is easy to check that an exact solution of the problem (5.1), (5.2) are the functions

$$
\left\{\begin{array}{l}
x_{1}^{*}=0.1 t \\
x_{2}^{*}=0.1 t^{2}
\end{array}\right.
$$

Suppose that the boundary-value problem (5.1), (5.2) is considered in the domain

$$
D=\left\{\left(x_{1}, x_{2}\right):\left|x_{1}\right| \leq 0.42,\left|x_{2}\right| \leq 0.4\right\} .
$$

Boundary conditions (5.2) can be rewritten in the form

$$
C x\left(\frac{1}{2}\right)+g\left(x(0), x\left(\frac{1}{4}\right) x\left(\frac{1}{2}\right)\right)=C x\left(\frac{1}{2}\right),
$$

where

$$
\begin{gathered}
C=I_{2}, \\
I_{2}=\left(\begin{array}{ll}
1 & 0 \\
0 & 1
\end{array}\right),
\end{gathered}
$$

$g\left(x(0), x\left(\frac{1}{4}\right) x\left(\frac{1}{2}\right)\right)=\operatorname{col}\left(g_{1}\left(x(0), x\left(\frac{1}{4}\right) x\left(\frac{1}{2}\right)\right), g_{2}\left(x(0), x\left(\frac{1}{4}\right) x\left(\frac{1}{2}\right)\right)\right)$.

Let us replace the values of the components of the solution of the boundary-value problem (5.1), (5.2) at the points $t=0, t=\frac{1}{4}$ and $t=\frac{1}{2}$ by parameters $z_{1}, z_{2}, \eta_{1}$, $\eta_{2}$ and $\lambda_{1}, \lambda_{2}$ :

$$
\begin{gathered}
z:=x(0)=\operatorname{col}\left(x_{1}(0), x_{2}(0)\right)=\operatorname{col}\left(z_{1}, z_{2}\right), \\
\eta:=x\left(\frac{1}{4}\right)=\operatorname{col}\left(x_{1}\left(\frac{1}{4}\right), x_{2}\left(\frac{1}{4}\right)\right)=\operatorname{col}\left(\eta_{1}, \eta_{2}\right), \\
\lambda:=x\left(\frac{1}{2}\right)=\operatorname{col}\left(x_{1}\left(\frac{1}{2}\right), x_{2}\left(\frac{1}{2}\right)\right)=\operatorname{col}\left(\lambda_{1}, \lambda_{2}\right) .
\end{gathered}
$$

Using (5.6), the boundary restrictions (5.5) can be rewritten as

$$
\begin{gathered}
x(0)=z, \\
x\left(\frac{1}{2}\right)=\lambda-g(z, \eta, \lambda) .
\end{gathered}
$$

Let us put

$$
d(z, \eta, \lambda):=\lambda-g(z, \eta, \lambda) .
$$

where $z, \eta$ and $\lambda$ are given by (5.6). 
Using (5.8), the parametrized boundary conditions (5.7) can be written in the form:

$$
\begin{gathered}
x(0)=z, \\
x\left(\frac{1}{2}\right)=d(z, \eta, \lambda) .
\end{gathered}
$$

It is easy to check that the matrix $K$ from the Lipschitz condition (4.3) is

$$
K=\left(\begin{array}{cc}
0 & 0.05 \\
0.5 & 1
\end{array}\right)
$$

and

when $T=\frac{1}{2}$.

$$
r(K)<1.03<\frac{10}{3 T}
$$

Vector $\delta_{D}(f)$ can be chosen as

$$
\delta_{D}(f) \leq\left(\begin{array}{c}
0.03125 \\
0.515
\end{array}\right)
$$

Domain $D_{\beta}$ is defined by inequalities:

$$
\begin{gathered}
\left|0.045+z_{2}^{2}\right| \leq 0.42 \\
\left|-z 1+z_{2}+0.025\right| \leq 0.4
\end{gathered}
$$

One can verify that, for the parametrized boundary-value problem in this example, all of the necessary conditions are fulfilled. So, we can proceed with the application of the numerical-analytic scheme described above and thus construct the sequence of approximate solutions.

The components of the iteration sequence (4.5) for the boundary-value problem (5.1) under the linear parametrized two-point boundary conditions (5.9) have the form

$$
\begin{array}{r}
x_{m, 1}(t, z, \eta, \lambda):=z_{1}+\int_{0}^{t} f_{1}\left(s, x_{m-1,1}(s, z, \eta, \lambda), x_{m-1,2}(s, z, \eta, \lambda)\right) d s- \\
-2 t \int_{0}^{\frac{1}{2}} f_{1}\left(s, x_{m-1,1}(s, z, \eta, \lambda), x_{m-1,2}(s, z, \eta, \lambda)\right) d s+ \\
+2 t\left(z_{2}^{2}+\eta_{1}+0.025-z_{1}\right), \\
x_{m, 2}(t, z, \eta, \lambda):=z_{2}+\int_{0}^{t} f_{2}\left(s, x_{m-1,1}(s, z, \eta, \lambda), x_{m-1,2}(s, z, \eta, \lambda)\right) d s- \\
\left.-2 t \int_{0}^{\frac{1}{2}} f_{2}\left(s, x_{m-1,1}(s, z, \eta, \lambda), x_{m-1,2}(s, z, \eta, \lambda)\right)\right) d s+ \\
+2 t\left(0.025-z_{1}\right),
\end{array}
$$

where $m=1,2,3, \ldots$,

$$
x_{0,1}(t, z, \eta, \lambda)=z_{1}+2 t\left(z_{2}^{2}+\eta_{1}+0.025-z_{1}\right),
$$




$$
x_{0,2}(t, z, \eta, \lambda)=z_{2}+2 t\left(0.025-z_{1}\right) .
$$

The system of approximate determining equations (4.53)-(4.55) depending on the number of iterations for the given example is

$$
\begin{array}{r}
\Delta_{m, 1}(z, \eta, \lambda)=-2 \int_{0}^{\frac{1}{2}} f_{1}\left(s, x_{m-1,1}(s, z, \eta, \lambda), x_{m-1,2}(s, z, \eta, \lambda)\right) d s+ \\
+2\left(z_{2}^{2}+\eta_{1}+0.025-z_{1}\right)=0, \\
\Delta_{m, 2}(z, \eta, \lambda)=-2 \int_{0}^{\frac{1}{2}} f_{2}\left(s, x_{m-1,1}(s, z, \eta, \lambda), x_{m-1,2}(s, z, \eta, \lambda)\right) d s+ \\
+2\left(0.025-z_{1}\right)=0 \\
x_{m, 1}\left(\frac{1}{4}, z, \eta, \lambda\right)=\eta_{1}, x_{m, 2}\left(\frac{1}{4}, z, \eta, \lambda\right)=\eta_{2}, \\
x_{m, 1}\left(\frac{1}{2}, z, \eta, \lambda\right)=\lambda_{1}, x_{m, 2}\left(\frac{1}{2}, z, \eta, \lambda\right)=\lambda_{2} .
\end{array}
$$

Using (5.10)-(5.13), as a result of the first iteration $(m=1)$, by Maple 13 we get:

$$
\begin{gathered}
x_{11}=z_{1}-0.001666666667 t^{3}+0.00125 t^{2}-0.05 t^{2} z_{1}+ \\
+0.04979166666 t-1.975 t z_{1}+2 t z_{2}^{2}+2 t \eta_{1} \\
x_{12}=z_{2}+0.002 t^{5}-0.0008333333332 t^{3}+0.06666666666 t^{3} z_{1}- \\
-1.333333333 t^{3} z_{1}^{2}+0.5 t^{2} z_{2}^{2}+0.5 t^{2} \eta_{1}+0.875 t^{2}-0.5 t^{2} z_{1}- \\
-0.05 t^{2} z_{2}+2 t^{2} z_{2} z_{1}-1.766666667 t z_{1}-0.25 t z_{2}^{2}+ \\
+0.006333333333 t+0.3333333334 t z_{1}^{2}-0.25 t \eta_{1}+ \\
+0.025 z 2 t-t z_{2} z_{1},
\end{gathered}
$$

for all $t \in\left[0, \frac{1}{2}\right]$.

The system (5.14)-(5.17), after the first iteration, has the form

$$
\begin{gathered}
\Delta_{1,1}(z, \eta, \lambda):=-0.0500263021-1.975937500 z_{1}- \\
-0.002083333334 z_{1}^{2}+2.001041667 \eta_{1}+0.004166666666 z_{2} z_{1}- \\
-0.05010416666 z_{2}+2.001041667 z_{2}^{2}=0 \\
\Delta_{1,2}(z, \eta, \lambda):=0.1631250001 z_{2} z_{1}^{2}-0.04177083332 z_{2} \eta_{1}- \\
-0.1471874999 z_{2}^{2} z_{1}-0.002083333338 z_{1}^{2} \eta_{1}-000.8333333351 z_{1}^{3} z_{2}+ \\
+0 .--6250000002 z_{1}^{2} z_{2}^{2}+0.0005208333338 \eta_{1}^{2}+0.01989583334 \eta_{1} z_{1}+ \\
+0.001041666668 \eta_{1} z_{2}^{2}+0.00416666667 z_{2}^{3} z_{1}-0.04177083332 z_{2}^{3}+ \\
+0.002116402121 z_{1}^{4}-0.04257275137 z_{1}^{3}+0.0005208333338 z_{2}^{4}+ \\
+0.006258188451+0.004166666670 \eta_{1} z_{2} z_{1}+0.3158912369 z_{1}^{2}- \\
-0.9658448663 z_{2} z_{1}-2.263765001 z_{1}+0.01775263207 z_{2}- \\
-0.2503388207 \eta_{1}+0.7538330543 z_{2}^{2}=0 \\
0.503125 z_{1}+0.01249999999+0.5 z_{2}^{2}+0.5 \eta_{1}=\eta_{1}
\end{gathered}
$$




$$
\begin{gathered}
1.003125 z_{2}+0.007041015625-0.4718750001 z_{1}+0.06250000002 z_{1}^{2}- \\
-0.03125 z_{2}^{2}-0.03125 \eta_{1}-0.125 z_{2} z_{1}=\eta_{2} .
\end{gathered}
$$

The computation shows that the approximate solutions of the approximate determining system (5.18)-(5.21) are

$$
\begin{gathered}
z_{1}:=z_{11}=-1.732102940 \cdot 10^{-8}, \\
z_{2}:=z_{12}=-0.000005209304726, \\
\eta_{1}:=\eta_{11}=0.02499998258 \\
\eta_{2}:=\eta_{12}=0.006254548758 \\
\lambda_{1}:=\lambda_{11}=0.0499999826 \\
\lambda_{2}:=\lambda_{12}=0.02499480802 .
\end{gathered}
$$

The first approximation for the first and second components of the solution is

$$
\begin{aligned}
& x_{11}=-1.73210294 \cdot 10^{-8}-0.001666666667 t^{3}+ \\
&+ 0.001250000866 t^{2}+0.09979166608 t, \\
& x_{12}=-0.000005209304726+0.002 t^{5}-0.0008333344879 t^{3}+ \\
&+ 0.1000002604 t^{2}+0.00008323804922 t .
\end{aligned}
$$

The graphs of first approximation and the exact solution of the original boundaryvalue problem are shown on Figure 1. The error of the first approximation is
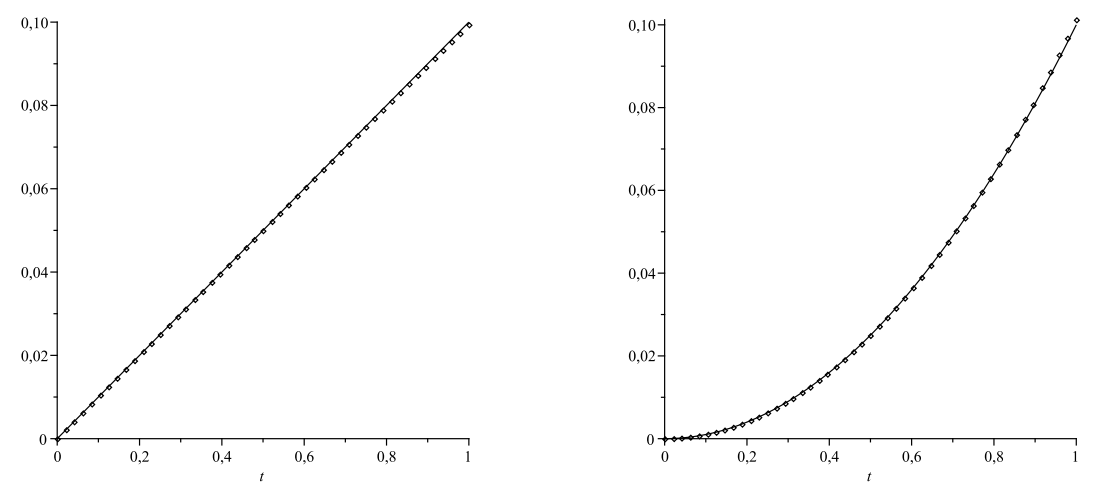

FIgURE 1. The first components of the exact solution (solid line) and its first approximation (drawn with dots)

$$
\begin{gathered}
\max _{t \in\left[0, \frac{1}{2}\right]}\left|x_{1}^{*}(t)-x_{11}(t)\right| \leq 1.0041 \cdot 10^{-5}, \\
\max _{t \in\left[0, \frac{1}{2}\right]}\left|x_{2}^{*}(t)-x_{12}(t)\right| \leq 6,8 \cdot 10^{-6} .
\end{gathered}
$$


The error of the third approximation is

$$
\begin{aligned}
& \max _{t \in\left[0, \frac{1}{2}\right]}\left|x_{1}^{*}(t)-x_{31}(t)\right| \leq 1.51 \cdot 10^{-9}, \\
& \max _{t \in\left[0, \frac{1}{2}\right]}\left|x_{2}^{*}(t)-x_{32}(t)\right| \leq-1.264 \cdot 10^{-9} .
\end{aligned}
$$

Continuing our iterations we will get more precise approximate solutions of the original boundary-value problem.

\section{ACKNOWLEDGEMENT}

This research was carried out as part of the TAMOP-4.2.1.B-10/2/KONV-20100001 project supported by the European Union and co-financed by the European Social Fund.

\section{REFERENCES}

[1] K. Marynets, "On the parametrization for nonlinear boundary value problems with nonlinear boundary conditions," Miskolc Math. Notes, vol. 12, no. 2, pp. 209-223, 2011.

[2] K. V. Marynets', "Investigation of a Cauchy-Nicoletti type three point boundary value problem," Visn., Ser. Fiz.-Mat. Nauky, Kyï. Univ. Im. Tarasa Shevchenka, vol. 2009, no. 3, pp. 85-90, 2009.

[3] A. Rontó and M. Rontó, "On the investigation of some boundary value problems with nonlinear conditions," Math. Notes, Miskolc, vol. 1, no. 1, pp. 43-55, 2000.

[4] A. Rontó and M. Rontó, "On a Cauchy-Nicoletti type three-point boundary value problem for linear differential equations with argument deviations," Miskolc Math. Notes, vol. 10, no. 2, pp. 173-205, 2009.

[5] A. N. Rontó, M. Rontó, and N. M. Schobak, "Parametrization of three-point nonlinear boundary value problems," Nelinijni Kolyvannya, vol. 7, no. 3, pp. 395-413, 2004.

[6] M. Rontó and J. Mészáros, "Some remarks concerning the convergence of the numerical-analytic method of successive approximation," Ukr. Math. J., vol. 48, no. 1, pp. 101-107, 1996.

[7] M. Rontó and A. M. Samoilenko, Numerical-analytic methods in the theory of boundary-value problems. Singapore: World Scientific, 2000.

Authors' addresses

Miklós Rontó

Department of Analysis, University of Miskolc, 3515, Miskolc Egyetemváros

E-mail address: matronto@uni-miskolc.hu

\section{K. Marynets}

Uzhhorod National University, Ukraine

E-mail address: katya_marinets@ukr.net 